\title{
INTERPRETATION OF INFRARED VIBRATION-ROTATION SPECTRA OF INTERSTELLAR AND CIRCUMSTELLAR MOLECULES
}

\author{
JOHN H. LACY \\ Department of Astronomy, University of Texas, Austin, TX 78712, USA; lacy@astro.as.utexas.edu \\ Received 2012 October 16; accepted 2013 January 22; published 2013 February 26
}

\begin{abstract}
Infrared vibration-rotation lines can be valuable probes of interstellar and circumstellar molecules, especially symmetric molecules, which have no pure rotational transitions. But most such observations have been interpreted with an isothermal absorbing slab model, which leaves out important radiative transfer and molecular excitation effects. A more realistic non-LTE and non-isothermal radiative transfer model has been constructed. The results of this model are in much better agreement with the observations, including cases where lines in one branch of a vibration-rotation band are in absorption and another in emission. In general, conclusions based on the isothermal absorbing slab model can be very misleading, but the assumption of LTE may not lead to such large errors, particularly if the radiation field temperature is close to the gas temperature.
\end{abstract}

Key words: ISM: molecules - line: formation - radiative transfer

\section{THE PROMISE}

Infrared spectroscopy of molecular vibration-rotation lines holds the promise of providing a valuable probe of chemical and physical conditions in interstellar and circumstellar gas. Vibration-rotation spectra complement pure rotational spectra in several important ways. First, molecules without permanent dipole moments, such as $\mathrm{CH}_{4}, \mathrm{C}_{2} \mathrm{H}_{2}$, and $\mathrm{CO}_{2}$, can be observed when vibration breaks the symmetry of the molecule. Second, because vibration-rotation lines are typically seen in absorption toward embedded young stars, they selectively probe gas in starforming regions. And third, because vibration-rotation lines of different rotational states lie close together in wavelength, they can be observed with one instrument and telescope, and often simultaneously.

It seems that the interpretation of absorption lines should be straightforward. The optical depth of an absorption line depends primarily on the column density in the lower state, that is, $N_{\mathrm{J}}(v=0)$, so the calculation of $N_{\mathrm{J}}$ from the spectrum is simply a matter of dividing the equivalent width of a line by the line-strength factor obtained from laboratory spectroscopy. If many lines are observed, it is not even necessary to assume a thermal distribution of lower-state populations, as the population of each rotational state can be measured. Of course, for optically thick lines curve of growth or other corrections for saturation may be necessary. This introduces uncertainties due to the generally unknown line shape, but the problem can be avoided if necessary by observing optically thin lines of rare isotopes. This interpretation procedure is referred to as the isothermal absorbing slab model. Variations on it have been used by astronomers to interpret almost all infrared molecular absorption spectra.

\section{THE PROBLEMS}

There are several problems with the isothermal absorbing slab model: the dust that emits the "background" continuum radiation is often mixed with the absorbing gas, the molecular gas emits as well as absorbs radiation, and there is a temperature gradient in the gas and dust around embedded sources.

As a first approximation, the effect of mixed gas and dust can be taken into account by considering the derived molecular column densities to be the columns to a depth in the source where the dust optical depth, $\tau$, reaches 1 . But since the dust opacity is wavelength dependent, this depth generally differs for different molecules or different vibration-rotation bands and may even differ substantially for lines within a band. In addition, the emergent continuum radiation at a given wavelength is not necessarily emitted from near $\tau=1$. If there is a temperature gradient in the dust, with the dust in the source being hotter at greater depth, emission by dust at $\tau>1$ may dominate the outgoing radiation, especially at wavelengths shortward of the peak of the Planck function. Without a radiative transfer calculation that takes these effects into account, the column of gas through which absorption-line observations are made may be substantially underestimated, and even derived molecular abundance ratios may be in error.

The effect of emission in the molecular lines is even more difficult to determine and account for. If a source is spatially resolved and the molecular gas is in LTE at a known temperature, the equation of radiative transfer along the line of sight is easily solved. The result is that molecular lines saturate at an intensity equal to the Planck function at the line wavelength. But often the continuum source is unresolved and the absorbing and emitting gas may have different angular extents, which may not fall entirely within the observing beam. Even more problematical is the fact that collisional deexcitation cross sections are small (Gonzalez-Alfonso et al. 2002) and radiative rates are relatively large for vibration-rotation transitions, making the critical densities for vibrational thermalization large, typically $>10^{12} \mathrm{~cm}^{-2}$. Consequently, vibrational LTE at the gas kinetic temperature could be a very poor approximation. It may be a better approximation to assume that all radiative excitations are followed by radiative decay. In a spherically symmetric situation in which the molecules lie in a shell separated from the continuum source, but within the observing beam, there may be no net absorption even if the lines are optically thick, since an equal number of photons are emitted into the beam as are absorbed out of the line of sight. The actual net absorption or emission depends on whether more or less molecular gas lies along the observing line of sight than along a typical direction and whether reemitted photons are absorbed by dust before escaping from the source region. In the case of sources with systematic motions, notably expanding shells around evolved 
stars, the emission and absorption may be separated spectrally by the Doppler effect. In those cases high spectral resolution observations would avoid this problem, but in many other cases the systematic motions are smaller than turbulent line widths or are unresolved, making it impossible to know how much the emission and absorption cancel.

In many cases the radiative transfer effects we have been discussing change the strengths of lines, thus leading to errors in derived abundances, but are not obvious from inspection of the spectra. But in a few cases there are recognizable symptoms of these effects. The importance of emission in vibrationrotation lines is clearest in the spectra of expanding circumstellar shells, with IRC +10216 being a particularly good example. The bending-mode $\mathrm{Q}$ branches of $\mathrm{HCN}$ and $\mathrm{C}_{2} \mathrm{H}_{2}$ near $14 \mu \mathrm{m}$ are prominent in the $R=2000$ ISO SWS spectrum of IRC+10216 (Cernicharo et al. 1999), with depths of $\sim 20 \%$ of the continuum, but the $\mathrm{P}$ and $\mathrm{R}$ branch regions of the spectrum show little evidence of line absorption or depression of the continuum due to lines. In contrast, the $R=80,000$ TEXES observations of Fonfría et al. (2008) show the R-branch lines of $\mathrm{HCN}$ and $\mathrm{C}_{2} \mathrm{H}_{2}$ to have typical depths of $\sim 50 \%$. The reason the lines are so much weaker in the ISO SWS spectrum is that the lines have P Cygni profiles, with nearly equal emission and absorption components that are blended together in the $R=2000$ spectrum. Apparently most vibrational excitations caused by absorption of photons in these bands are followed by emission, and most emitted photons escape from the shell, perhaps after multiple absorption and emission events.

The $13.7 \mu \mathrm{m} \nu_{5}$ band of $\mathrm{C}_{2} \mathrm{H}_{2}$ has also been observed toward a number of embedded high-mass young stars (Lacy et al. 1989; Evans et al. 1991; Lahuis \& van Dishoeck 2000; Knez et al. 2009; Barentine \& Lacy 2012). Evans et al. (1991) and Barentine $\&$ Lacy (2012) also observed absorption in the $7.5 \mu \mathrm{m} v_{4}+v_{5}$ band toward OMC1 IRc2 and NGC 7538 IRS 9. An energy level diagram of $\mathrm{C}_{2} \mathrm{H}_{2}$ with the relevant vibrational levels is shown in Figure 1. Both bands involve absorption from the ground vibrational level, but the derived $\mathrm{C}_{2} \mathrm{H}_{2}$ column density from the $v_{4}+v_{5}$ lines is greater than that derived from the $v_{5}$ lines by a factor of 3-10 toward IRc2 and 20 toward IRS 9. Both of the effects described above may contribute to this discrepancy. The dust opacity at $13.7 \mu \mathrm{m}$ is about twice as large as that at $7.5 \mu \mathrm{m}$, because of the wings of the 9.7 and $18 \mu \mathrm{m}$ silicate features. In addition, $7.5 \mu \mathrm{m}$ is farther off of the peak of the dust thermal emission, also causing the continuum to be formed farther into the sources (where the temperature is higher) at $7.5 \mu \mathrm{m}$. The amount of reemission in spectral lines may also differ between the $v_{5}$ and the $v_{4}+v_{5}$ bands, since the $v_{5}$ state can only decay to the ground via the $v_{5}$ band, whereas the $v_{4}+v_{5}$ state can decay either to the ground through emission of a $v_{4}+v_{5}$ photon or to the $v_{4}$ level by emission of a $v_{4}+v_{5}-v_{4}$ photon.

Ammonia $\left(\mathrm{NH}_{3}\right)$ also shows evidence of radiative transfer effects. Barentine \& Lacy (2012) observed lines in two branches of the $\mathrm{NH}_{3} v_{2}$ band toward NGC 7538 IRS 9. Although other molecules seen toward IRS 9, including $\mathrm{C}_{2} \mathrm{H}_{2}$ and $\mathrm{HCN}$, are seen in absorption and the aQ branch of $\mathrm{NH}_{3}$ shows $\mathrm{P}$ Cygni lines or weak absorption, aP- and sP-branch $\mathrm{NH}_{3}$ lines are seen in emission. Barentine \& Lacy (2012) briefly discuss a radiative transfer model to explain these observations. It assumes that the $\mathrm{NH}_{3}$ molecules are exposed to optically thin silicate emission, which is stronger on the $\mathrm{NH}_{3} \mathrm{R}$ and $\mathrm{Q}$ branches and thus causes more upward transitions in those branches than in the $\mathrm{P}$ branch. Since downward transitions are about equally likely in the three branches, emission dominates over absorption in the

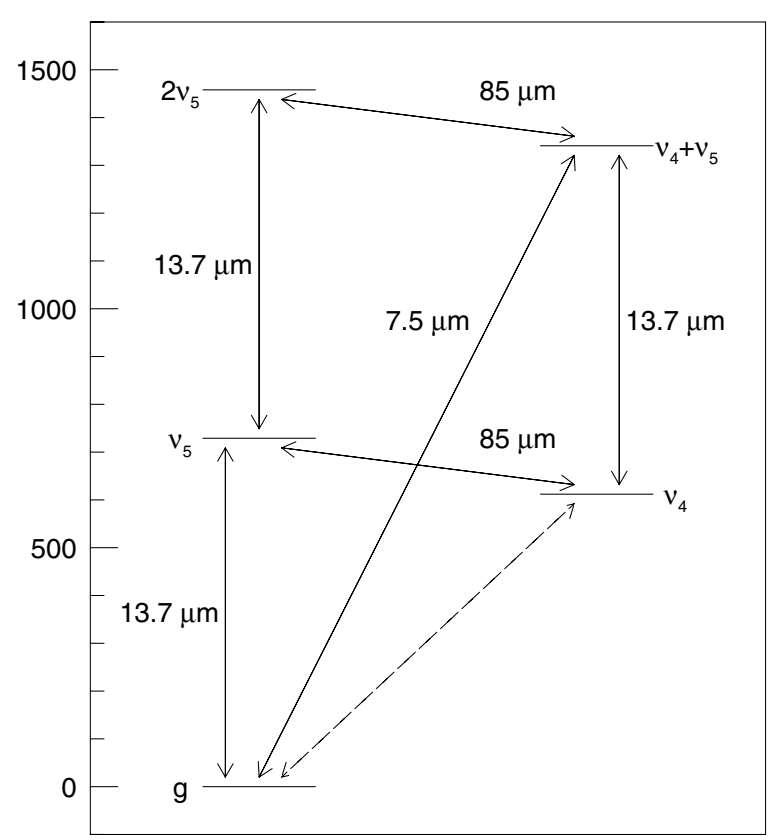

Figure 1. $\mathrm{C}_{2} \mathrm{H}_{2}$ vibrational energy levels and transitions of interest. The $v_{4}$ and $v_{5}$ modes are the anti-symmetric and symmetric bending modes, respectively. Allowed radiative transitions are indicated with solid arrows; the $v_{4}$ transition that only occurs collisionally is indicated with a dashed arrow. Allowed radiative transitions between these levels must change the quantum number $v_{5} ; v_{4}$ can change only if $v_{5}$ also changes. The $85 \mu \mathrm{m} v_{5}-v_{4}$ transitions have not been observed. Rotational levels and level splitting are not shown. The vertical scale is in $\mathrm{cm}^{-1}$.

$\mathrm{P}$ branch, and absorption dominates over emission in the $\mathrm{Q}$ and $\mathrm{R}$ branches.

\section{A RADIATIVE TRANSFER MODEL}

To reproduce and understand the radiative transfer and radiative excitation effects in molecular vibration-rotation spectra, I constructed a computer model that includes collisional and radiative excitation and deexcitation of molecules and calculates the outgoing spectrum.

The model assumes a parameterized distribution and composition of gas and dust in a shell around a blackbody luminosity source. The input parameters can be adjusted through a grid search procedure to fit the continuum and line spectra of individual sources. For the calculations in this paper we assume a spherically symmetric expanding shell. The model first calculates the dust temperature and continuum radiation field by alternately solving the equation of radiative transfer and the equation of thermal equilibrium for the dust. The radiative transfer calculation involves following a grid of rays through the shell. Typically, a $64 \times 64$ ray grid is used at the outer edge of the model. After following the rays halfway into the star, the inner $32 \times 32$ rays are split into $64 \times 64$ rays to increase the resolution. This is done up to 11 times, and then the rays are rejoined going out from the star. Rays that overlap the star are replaced by an appropriate fraction of the stellar spectrum before continuing out through the shell. As the rays are followed through the shell, the ray spectrum is added to a mean intensity spectrum stored for each grid cell, which is used to calculate the dust heating. The dust temperatures are calculated for cells on a spherical coordinate grid. For each grain type and size the equilibrium temperature is calculated that balances radiative heating and cooling. The dust source function is then 
recalculated and the radiative transfer calculation repeated. Typically 16 iterations of the radiative transfer and thermal equilibrium calculations are sufficient for even very optically thick dust shell models to converge. The gas temperature is not calculated separately but is assumed to equal the dust temperature. For the models described in this paper, the dust shell and stellar parameters were chosen to roughly reproduce the observed spectral energy distribution of NGC 7538 IRS 9.

After solving for the shell thermal structure, the program solves for the molecular level populations and the outgoing line spectrum. This is done by alternately solving the equation of radiative transfer in molecular lines and the equation of statistical equilibrium involving collisional and radiative rates in and out of vibrationally excited energy levels. Line strengths are calculated from band strengths and Hönl-London factors, as in Evans et al. (1991), and are consistent with those in the HITRAN database. The calculation is simplified for linear and symmetric top molecules by the selection rules that cause each vibrationally excited rotational level to be coupled radiatively to only two or three rotational levels in the ground vibrational state. It is further simplified by assuming that the rotational levels in the ground vibrational state are in LTE at the gas temperature. This and other assumptions are discussed in Section 5. We also assume that collisional transitions between vibrational states do not change the rotational state. This is not actually valid, but since collisions must drive populations toward LTE and the ground vibrational state is assumed to be in LTE, on average collisions should not change the rotational excitation of molecules. In addition, vibration-changing collisional transitions are relatively unimportant compared to radiative transitions at the densities found in the model. With these simplifications, the excited vibration-rotation level populations and the vibration-rotation lines between them and the ground vibrational state can be calculated for one excited vibration-rotation level at a time, neglecting any coupling between rotational levels of the excited vibrational state.

As with the dust calculation, the code follows a grid of rays through the shell, in this case calculating the spectrum for wavelengths close to the relevant vibration-rotation lines. At each step through the shell the molecular line profile is Doppler shifted by the component of any assumed systematic motion along the ray direction. As the rays propagate through the shell, the Dopplershifted spectrum is added to a sum for the rays passing through each spherical coordinate cell, so that the mean intensity, averaged over each spectral line, can be calculated for each cell. With the mean intensities, the radiative excitation and deexcitation rates are calculated, and with those and the collisional rates, the vibration-rotation level populations are calculated. The radiative transfer and level population calculations are then repeated until they converge. However, the fact that the molecular lines are typically more optically thick than the dust continuum results in slower convergence than for the calculation of the thermal structure. This problem was overcome by comparing the change in level populations in each iteration with that in the previous iteration and amplifying the correction if iterations are well correlated. With the accelerated convergence scheme, the level populations almost always converge within 16 iterations.

\section{RESULTS OF MODELING}

Models were run with parameters meant to roughly reproduce the IRS 9 observations. The central source was taken to be a $12,000 \mathrm{~K}, 10^{4} L_{\odot}$ blackbody. The gas and dust shell had a constant $\mathrm{H}_{2}+\mathrm{He}$ density of $2 \times 10^{7} \mathrm{~cm}^{-3}$ from 4 to $400 \mathrm{AU}$ and

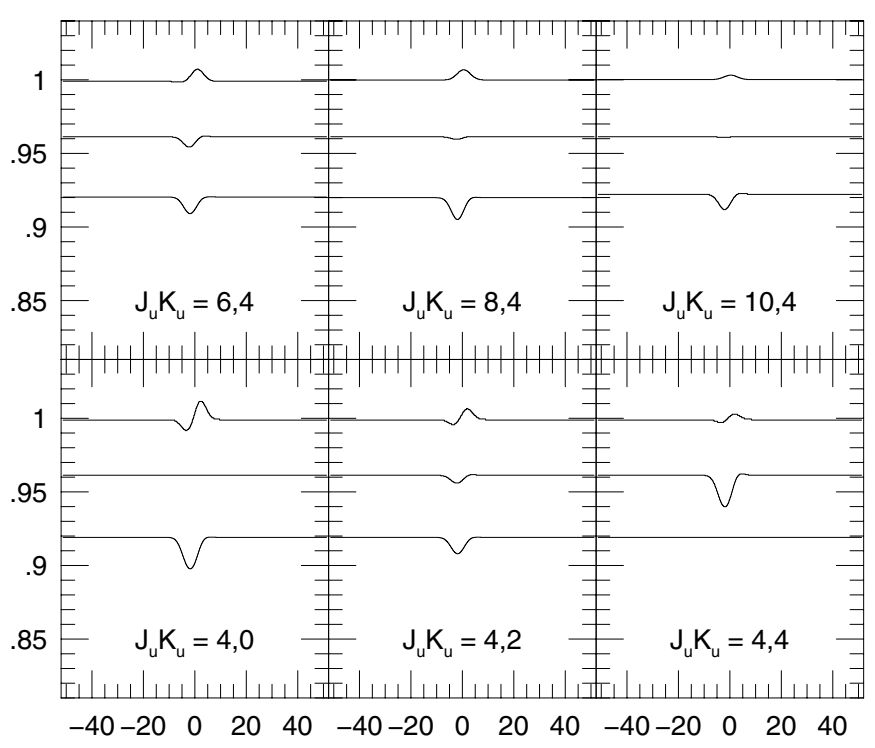

Figure 2. Model $\mathrm{NH}_{3} v_{2}$ umbrella-mode line profiles. In each panel, the P-, $\mathrm{Q}-$, and R-branch lines between a given J, K state in the excited vibrational level and the ground are shown (top to bottom). Spectra are normalized, with the Q- and R-branch lines offset downward. Q-branch lines are strongest for $\mathrm{K}_{u}=$ $\mathrm{J}_{u}$ and are absent for $\mathrm{K}_{u}=0$. R-branch lines are absent for $\mathrm{K}_{u}=\mathrm{J}_{u}$.

then fell with an $r^{-2}$ density profile out to $1.6 \times 10^{4} \mathrm{AU}$. Its mass was 4.2 $M_{\odot}$, and its radial column density was $1.8 \times 10^{23} \mathrm{~cm}^{-2}$. The calculated dust temperature was $1800 \mathrm{~K}$ at the inner edge of the shell, falling to $160 \mathrm{~K}$ at $400 \mathrm{AU}$ and $40 \mathrm{~K}$ at the outer edge of the shell. A constant expansion speed of $2 \mathrm{~km} \mathrm{~s}^{-1}$ and a turbulent velocity dispersion of $3 \mathrm{~km} \mathrm{~s}^{-1}$ were assumed. Molecular line profiles were calculated for the $\mathrm{C}_{2} \mathrm{H}_{2} \quad v_{5}$ and $v_{4}+v_{5}$ bands, with $\mathrm{C}_{2} \mathrm{H}_{2} / \mathrm{H}_{2}=1 \times 10^{-7}$, the $\mathrm{NH}_{3} v_{2}$ band, with $\mathrm{NH}_{3} / \mathrm{H}_{2}=4 \times 10^{-6}$, and the $\mathrm{CO} v=1-0$ band, with ${ }^{13} \mathrm{CO} / \mathrm{H}_{2}=1 \times 10^{-6}$ and $\mathrm{C}^{18} \mathrm{O} / \mathrm{H}_{2}=2 \times 10^{-7}$. These parameters are not meant to be fitted values, but they produced line depths similar to those observed.

Several of the calculated $\mathrm{NH}_{3}$ line profiles are shown in Figure 2. In each panel, the P-, Q-, and R-branch lines between a given $\mathrm{J}, \mathrm{K}$ state in the excited vibrational level and the ground vibrational level are shown. Note that these are normally referred to as the $\mathrm{P}\left(\mathrm{J}_{u}+1, \mathrm{~K}\right), \mathrm{Q}\left(\mathrm{J}_{u}, \mathrm{~K}\right)$, and $\mathrm{R}\left(\mathrm{J}_{u}-1, \mathrm{~K}\right)$ lines, being labeled by their rotational levels in the lower vibrational state. For this "umbrella" vibrational mode, Q-branch lines have the largest optical depths for $\mathrm{K}=\mathrm{J}$, and $\mathrm{P}$ - and $\mathrm{R}$-branch lines are strongest for $\mathrm{K}=0$. The nuclear statistical weights favor lines with $K=3 n$ by a factor of two. In all cases, the model P-branch lines are predominantly in emission, the Q-branch lines show weak absorption, and the R-branch lines are more strongly in absorption. This is in agreement with the observed P- and Q-branch lines toward IRS 9. The R-branch lines have not been observed but are predicted to be seen in absorption, with depths of $1 \%-2 \%$.

Several calculated $\mathrm{C}_{2} \mathrm{H}_{2} v_{5}$ lines are shown in Figure 3, and $v_{4}+v_{5}$ lines are shown in Figure 4. For $\mathrm{C}_{2} \mathrm{H}_{2}$, rotational states in the ground vibrational level with $\mathrm{J}_{l}$ odd (ortho states) have nuclear statistical weights of 3 ; those with $\mathrm{J}_{l}$ even (para states) have nuclear statistical weights of 1 . Line-strength factors for P-, Q-, and R-branch lines are proportional to $\mathrm{J}_{u}, 2 \mathrm{~J}_{u}+1$, and $\mathrm{J}_{u}+1$, respectively. Combining these factors for the lines shown, all of which have $\mathrm{J}_{u}$ even and so $\mathrm{J}_{l}$ odd for the $\mathrm{P}$ - and R-branch lines, the P- and R-branch lines have similar line-strength factors, whereas Q-branch lines are weaker on average by a factor of 


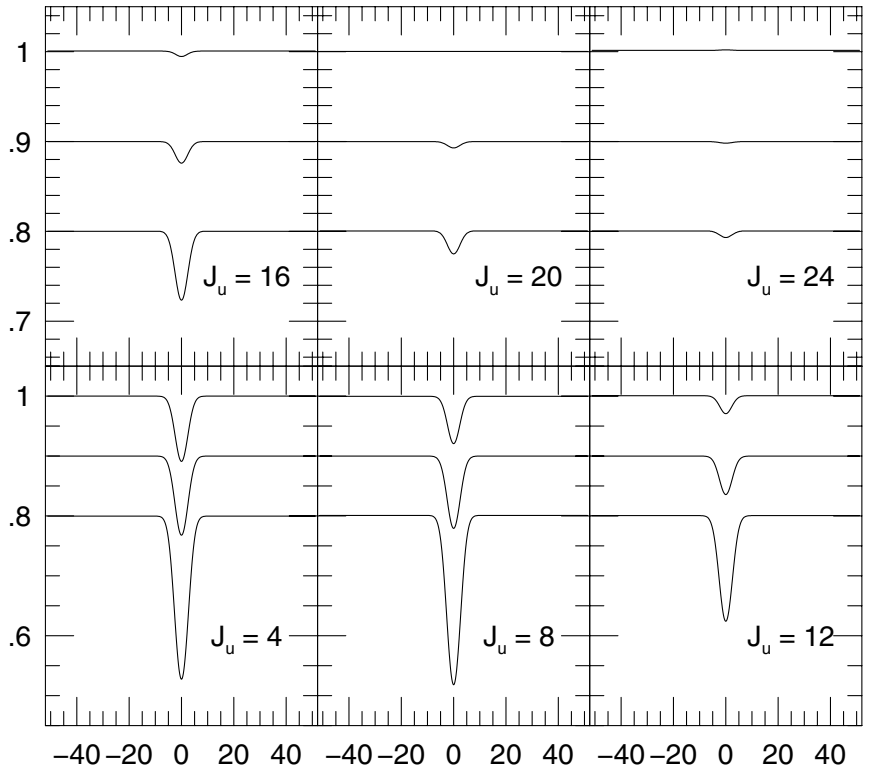

Figure 3. Model $\mathrm{C}_{2} \mathrm{H}_{2} v_{5}$ symmetric bending mode line profiles. In each panel, the P-, Q-, and R-branch lines between a given $\mathrm{J}_{u}$ level in the excited vibrational level and the ground are shown (top to bottom). Q- and R-branch lines are offset downward. Rotational levels of the excited vibrational level are split into ortho and para states, with the P-and R-branch lines going to the ortho $\left(\mathrm{g}_{n}=3\right)$ state for $\mathrm{J}_{u}$ even, and the Q-branch line going to the para $\left(\mathrm{g}_{n}=1\right)$ state.

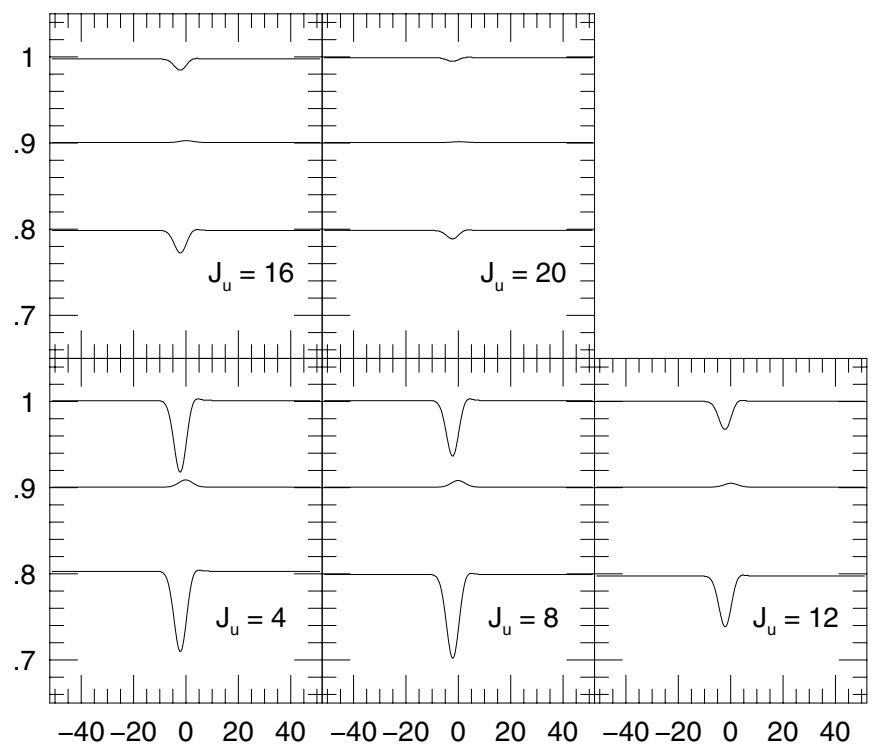

Figure 4. Model $\mathrm{C}_{2} \mathrm{H}_{2} v_{4}+v_{5}$ band line profiles. Only P-branch (top) and $\mathrm{R}$-branch (bottom) lines occur for this band; the middle spectrum is for the $v_{4}+v_{5}-v_{4}$ Q-branch line, which accounts for much of the radiative deexcitation of the $v_{4}+v_{5}$ state. Since it is scaled by the continuum flux, the $v_{4}+v_{5}-v_{4}$ line appears weaker than the $v_{4}+v_{5}$ lines.

$2 / 3$. In addition, for absorption lines the line-strength factors must be multiplied by the Boltzmann population factors of the lower rotational states.

In agreement with the IRS 9 observations, the $v_{5}$ R-branch lines have depths only about twice those of the $v_{4}+v_{5}$ lines, even though the $v_{5}$ band strength is nearly 10 times greater. The P-branch line depths are similar in the two bands. A new prediction is that the $v_{5} \mathrm{P}$-branch lines should be substantially weaker than the R-branch lines. At higher abundances the P-branch lines go into emission and the Q-branch lines show $\mathrm{P}$ Cygni profiles. The P-branch lines are unobservable from the

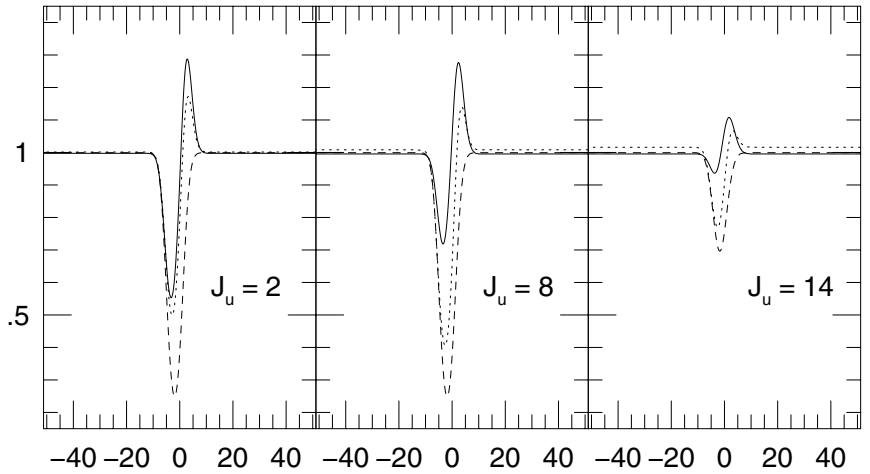

Figure 5. Model ${ }^{13} \mathrm{CO} v=1-0$ line profiles. Only P- (solid) and R-branch (dotted) lines occur for ${ }^{1} \Sigma$ diatomic molecules like $\mathrm{CO}$. The dashed line in each panel is for an average of the P- and R-branch line strengths, but leaving out emission in the line.

ground, due to telluric $\mathrm{CO}_{2}$ absorption. The Q-branch lines are difficult to observe, due to telluric absorption and blending of the closely spaced lines, but do show some evidence of P Cygni emission. The explanation of the strengths and profiles of these various lines is discussed below.

Calculated CO lines are shown in Figure 5. CO has no Q branch; the third line shown in each panel is for an average of the P- and R-branch line strengths, but with emission by molecules in the $v=1$ state turned off in the program, to show what would be observed if the pure-absorption model used in the past were valid. Both P- and R-branch lines of CO have P Cygni profiles, with the relative strength of the emission component being greater in the P-branch lines. The observed lines of $\mathrm{CO}$ from IRS 9 also have P Cygni profiles but are considerably broader than the lines of other molecules. This is probably a result of a high-velocity outflow with a different chemistry from the gas that dominates the spectra of other molecules. The high optical depths of the ${ }^{12} \mathrm{CO}$ and ${ }^{13} \mathrm{CO}$ lines may also contribute to the prominence of line wings that are not apparent in spectra of other molecules. $\mathrm{C}^{17} \mathrm{O}$ and $\mathrm{C}^{18} \mathrm{O}$ lines are blended with ${ }^{12} \mathrm{CO}$ and ${ }^{13} \mathrm{CO}$ lines in the existing observations; further observations would be desirable.

\section{EXPLANATION AND DISCUSSION}

The model spectra are generally in at least qualitative agreement with the observations of IRS 9. In particular, they reproduce the emission seen in $\mathrm{NH}_{3} \mathrm{P}$-branch lines and the similar depths of $\mathrm{C}_{2} \mathrm{H}_{2} \quad v_{5}$ and $v_{4}+v_{5}$ lines. However, they make additional predictions not anticipated by the simple radiative transfer models of Barentine \& Lacy (2012). Notably, they predict that $\mathrm{C}_{2} \mathrm{H}_{2}$ should show differences between its $\mathrm{P}-$, Q-, and R-branch lines like those seen in $\mathrm{NH}_{3}$. Somewhat less prominent effects are predicted for $\mathrm{CO}$. The explanation given by Barentine \& Lacy (2012) for $\mathrm{NH}_{3}$ was based on the brighter continuum radiation at the $\mathrm{Q}$ - and R-branch wavelengths. But this should not be the case for $\mathrm{C}_{2} \mathrm{H}_{2}$, for which the dust opacity is similar at the wavelengths of its different branches.

To help understand the radiative transfer effects responsible for the line profiles of different lines, several physical effects were changed in the model. First, a model was run assuming LTE populations of the excited vibration-rotation levels. Perhaps surprisingly, the model results did not change greatly; P-branch lines still favored emission relative to R-branch lines. Apparently the non-LTE populations were not very different from LTE populations. The explanation for this may be that 
the continuum radiation field seen by the molecules is not very different from a blackbody field at the dust temperature, and the gas temperature was assumed to be equal to the dust temperature. As a result, populations controlled by radiation were not very different from populations controlled by collisions. This would not be the case if the molecular gas were more distant from the warm dust responsible for the continuum, so the conclusion that non-LTE effects were relatively small for the IRS 9 model may not be valid in general. A second modification to the physics of the model was to set the rotational constant $\left(B_{e}\right)$ of the molecules to zero, so that the P-, Q-, and R-branch lines all fell at the same wavelength, and their lower-state energies were all the same. The differences between P-, Q-, and R-branch lines then disappeared. Finally, a model was run with the gas and dust temperature held constant throughout the shell. Again, the differences between P-, Q-, and R-branch lines largely disappeared. The conclusion from these models with modified physics was that the difference between the lower-state energies and the associated Boltzmann factors was the primary cause of the different line profiles.

With the results of the modified models, the explanation for the differences between the P-, Q-, and R-branch lines can be understood. We will consider P- and R-branch lines, which have similar behaviors for all molecules. (The strengths of Q-branch lines depend on the molecular symmetry and vibrational mode.) The P- and R-branch lines from a given rotational state of the upper vibrational level have nearly equal Einstein $A$ coefficients (depending weakly on $\mathrm{J}_{u}$ ) and thus nearly equal branching ratios on emission. However, the absorption in these lines depends on the radiation field and the populations of the rotational states of the lower vibrational level. Especially for high values of $\mathrm{J}$, these populations can differ substantially, because of the different energy levels of $\mathrm{J}_{l}=\mathrm{J}_{u}+1$ and $\mathrm{J}_{l}=\mathrm{J}_{u}-1$ for the $\mathrm{P}$ - and R-branch lines, respectively. As a result, the two lines have similar emission strengths, but the R-branch line has greater absorption. If most upward transitions are followed by a downward radiative transition, emission and absorption nearly cancel, but with emission dominating in the P-branch line and absorption dominating in the R-branch line. In the case of $\mathrm{NH}_{3}$ the greater radiation field at the wavelengths of the $\mathrm{R}$-branch lines enhances the effect, but the effect is present even for $\mathrm{C}_{2} \mathrm{H}_{2}$ and $\mathrm{CO}$ in models with a temperature gradient through the shell.

The disappearance of the difference between P-and R-branch lines in the isothermal model can be explained by the fact that the continuum radiation field at the $\mathrm{R}$-branch wavelengths is weaker than that at the P-branch wavelengths for a given $\mathrm{J}_{u}$ by the same Boltzmann factor that the lower-level population is greater, resulting in approximately the same number of upward transitions in the two lines. The temperature gradient in the dust shell model caused the color temperature of the continuum radiation field to be higher than the gas temperature where the lines are formed. As a result, heat must flow from the radiation field into the gas. This happens by net absorption of R-branch photons and net emission of P-branch photons, pumping the rotational populations.

It would be very desirable to estimate the magnitude of the error that is made using the isothermal absorbing slab model. For the models shown here, R-branch lines are typically a factor $\sim 2$ weaker when reemission is included, but P-branch lines may be dominated by emission. Unfortunately, the extent to which emission cancels absorption depends on the probability that an emitted photon escapes, rather than being absorbed by dust, and the asymmetry of the source, which determines how the absorption along our line of sight compares to that along other lines of sight. An understanding of the geometry of the source being observed would be needed to make a realistic model including reemission.

Several simplifications and approximations made in the model should be noted. First, the gas temperature was assumed to be equal to the dust temperature. This assumption may be valid through much of the modeled shell, even if the density is too low for collisions with dust grains to dominate the heating and cooling of the gas, since the radiation field temperature is close to the dust temperature through most of the shell. However, close to the exciting star ultraviolet radiation may heat the gas to a higher temperature than the dust, via the photoelectric effect. The optical depth of the dust in the model is high enough to hide this region, but in the case of less deeply embedded objects, where the gas near the exciting source can be observed, departure of the gas temperature from the dust temperature could be important. To test this effect, a less optically thick model was run that included photoelectric heating of the gas and allowed the gas and dust temperature to differ. In this model, the gas temperature was greater than the infrared radiation temperature, so energy should flow from the gas into the radiation. In fact, P-branch lines then showed greater absorption than R-branch lines, as is expected by this thermodynamic argument.

Another assumption was that the rotational temperature of the molecules was equal to the gas temperature. This might be justified by the fact that the density in most of the shell is greater than the critical density for rotational thermalization, especially for symmetric molecules, like $\mathrm{C}_{2} \mathrm{H}_{2}$ and $\mathrm{CH}_{4}$, which have no allowed rotational transitions. However, the infrared pumping that led to differing P- and R-branch line profiles may modify the populations. The importance of infrared pumping can be estimated by comparing the rate of collisional transitions between rotational levels to the rate of infrared transitions between the ground and excited vibrational levels. The collisional transition rate is given by the product of the collisional rate coefficient, which is typically $\sim 10^{-10}$, and the gas density, giving a rate of $\sim 2 \times 10^{-3} \mathrm{~s}^{-1}$. The radiative rate is given by the product of the vibrational $A$ coefficient and the fractional population of the excited vibrational state. For $\mathrm{C}_{2} \mathrm{H}_{2}$, with an $A$ coefficient of $6 \mathrm{~s}^{-1}$ for the sum of the $\mathrm{P}$ - and R-branch lines to a given rotational state, infrared pumping should dominate for vibrational temperatures $>130 \mathrm{~K}$, or throughout much of the model shell. This calculation may overestimate the importance of infrared pumping since absorption followed by emission of a vibration-rotation photon can at most change the rotational quantum number by 2 , whereas large $\Delta \mathrm{J}$ can occur in collisional transitions. But if the rotational populations are controlled by infrared pumping, the rotational temperature should equal the infrared radiation color temperature, and the difference between P- and R-branch lines should disappear. The resolution of this problem may be that the density of the gas around IRS 9 is enough greater than that assumed in the model so that collisions, rather than infrared pumping, control the rotational level populations. A similar effect is the pumping of vibrational levels by radiative and collisional transitions to other vibrational states. For example, the $v_{5}$ state of $\mathrm{C}_{2} \mathrm{H}_{2}$ could be populated by collisional or radiative transitions from the $v_{4}$ state, which itself has several possible methods of excitation. To include the various neglected ways in which the excited vibration-rotation states can be populated would require the simultaneous calculation of many vibration-rotation 
states. Unfortunately, that is beyond the capability of the model used here.

\section{CONCLUSIONS}

The first conclusion of this work is that interpretation of vibration-rotation spectra based on the isothermal absorbing slab model can be very misleading. In general, column densities derived with this model can be expected to be underestimates, due to the neglect of reemission by molecules following absorption, but the magnitude of the error depends on the source geometry and the lines observed.

The second conclusion is more positive. Although collisions are not normally sufficiently frequent to maintain LTE at the gas kinetic temperature, the radiation field temperature may be similar to the gas temperature, and so molecular level populations may nevertheless be close to LTE populations. This was the case for the model considered and should generally be the case if the dust optical depth is large. As a result, non-LTE effects may not be large. Effects of radiative transfer through gas and dust with a temperature gradient are likely to be more important. Fortunately, these effects are easier to include in models used to interpret observations, and they should be included.

I thank the anonymous referee for several interesting comments, in particular pointing out the possibility of infrared pumping of the rotational populations.

\section{REFERENCES}

Barentine, J. C., \& Lacy, J. H. 2012, ApJ, 757, 111

Cernicharo, J., Yamamura, I., Gonzalez-Alfonso, E., et al. 1999, ApJ, 526, 41 Evans, N. J., II, Lacy, J. H., \& Carr, J. S. 1991, ApJ, 383, 674

Fonfría, J. P., Cernicharo, J., Richter, M. J., \& Lacy, J. H. 2008, ApJ, 673, 445 Gonzalez-Alfonso, E., Wright, C. M., Cernicharo, J., et al. 2002, A\&A, 386, 1074

Knez, C., Lacy, J. H., Evans, N. J., II, van Dishoeck, E. F., \& Richter, M. J. 2009, ApJ, 696, 471

Lacy, J. H., Evans, N. J., II, Achtermann, J. M., et al. 1989, ApJL, 342, L43

Lahuis, F., \& van Dishoeck, E. F. 2000, A\&A, 355, 699 